\title{
An analysis of community formation in faculty online learning communities
}

\author{
Alexandra Lau, ${ }^{1}$ Melissa H. Dancy, ${ }^{1}$ Joel C. Corbo, ${ }^{2}$ Charles Henderson, ${ }^{3}$ and Andy Rundquist ${ }^{4}$ \\ ${ }^{1}$ Department of Physics, University of Colorado, 390 UCB, Boulder, CO 80309 \\ ${ }^{2}$ Center for STEM Learning, University of Colorado, 393 UCB, Boulder, CO 80309 \\ ${ }^{3}$ Department of Physics, Western Michigan University, 1903 W. Michigan Ave., Kalamazoo, MI 49008 \\ ${ }^{4}$ Department of Physics, Hamline University, MS-C1913, 1536 Hewitt Ave., St. Paul, MN 55104
}

\begin{abstract}
Faculty Online Learning Communities (FOLCs) are designed to provide faculty support in the year following their attendance at the New Faculty Workshop. We hypothesize that membership in a community of faculty with shared interest in improving their teaching will lead to sustained adoption of research-based instructional strategies. We are not yet at the stage in our project where we can report on long-term effects of the FOLCs, but in this paper we present our initial analysis of the community that formed in each of our FOLC cohorts. Through interview data we show that most participants felt their FOLC was a community and we report on their reasons for feeling this way. We also discuss how the community was a support for the members' teaching efforts.
\end{abstract}

\section{INTRODUCTION}

Faculty Online Learning Communities (FOLCs) have been implemented to support faculty for the year following their attendance at the Physics and Astronomy New Faculty Workshop [1, NFW]. While the NFW is highly effective at motivating participants to try Research-Based Instructional Strategies (RBISs), attendees are just as likely as non-attendees to discontinue use of the RBIS they tried after facing situational barriers $[2,3]$. FOLCs were designed to better support sustained and productive adoption of strategies like those learned at the NFW.

Each FOLC cohort has one or two facilitators and around ten participants. The facilitators attend the NFW and recruit participants through informal interactions and a formal workshop session. The FOLCs run for the year following the NFW. Members meet biweekly via a video conference platform and connect in between meetings using an asynchronous communication platform similar to Facebook. Members choose the topics of the synchronous video meetings. Usually, an expert in some area of teaching is invited to speak with the cohort for part of the meeting, and in the other part members discuss their teaching as a group. During the second half of the FOLC, participants engage in a Scholarship of Teaching and Learning (SoTL) project in which they try something new in their teaching and assess the results [4]. For more details on the design of the FOLCs see [5].

Community is at the core of FOLCs. We are now halfway through the FOLC project and have collected enough data to conduct a preliminary analysis of the community that formed (or did not) in each cohort. In this paper we report on the results of this initial analysis which comes from participants' post-interviews.

\section{COMMUNITY-BASED MODEL OF CHANGE}

Community has many meanings. For this project we consider a successful community to have formed if the group members support each other around teaching by (1) learn- ing from each other by providing knowledge-based support (i.e. answering questions about a particular RBIS, sharing materials, etc.) and (2) providing affective support (i.e. encouragement to deal with frustrations, insecurities, etc.).

It is common for professors to have a research community, composed of people both inside and outside their university, with which they brainstorm ideas, trouble shoot problems, and gather feedback on their work. This network supports a faculty member as they explore new ideas and test new techniques. It is less common for professors to have such a community for the teaching aspect of their work [6].

Faculty Learning Communities (FLCs) were developed to support faculty as they endeavor to improve their teaching [4]. An underlying principle of FLCs is that as faculty work to transform their teaching, it is highly beneficial for them to pursue that work within a community. Formally, FLCs are defined as, "A cross-disciplinary faculty and staff group...who engage in an active, collaborative, yearlong program with a curriculum about enhancing teaching and learning" [4]. FLC members (who are all at the same university) meet in-person for seminars as well as community building activities. FLCs have spread to many institutions and have been shown to be effective at improving teaching and learning [4].

FOLCs were inspired by the FLC model, although they differ in two main ways: they are disciplinary (rather than interdisciplinary) and they are "virtual" (rather than in-person, at one university). Nonetheless, we designed the FOLCs with an explicit goal of promoting community formation. Every synchronous meeting has time reserved for the cohort to talk as a group, with no outside guest; during this time they often give a "state of the classroom" update or share a success or challenge from their teaching. This time was intentionally built into the meetings so the cohort could get to know each other and work through problems together. We also have the facilitators model being vulnerable by sharing one of their own teaching struggles and asking the cohort for feedback; this is meant to build trust among the cohort. Additionally, we chose to have an asynchronous communication platform so the cohort could communicate between their synchronous meetings. We hoped this would help the cohort stay connected and continue conversations that were seeded during 
their synchronous meetings.

We hypothesize that FOLCs will result in improved teaching and sustained adoption of RBISs. We cannot say whether these outcomes are achieved until we collect longitudinal data. However, our current data does help us see how participants are supporting each other and if participants value this support. Specifically, in this paper we use participants' self-reported experiences of the FOLC to answer (1) Did participants feel a community formed? What is the basis for their assessment? and (2) Did they feel others were a support for their teaching efforts?

\section{PARTICIPANTS AND METHODS}

To date, four cohorts have completed their FOLC experience. The first cohort ran for one semester, while Cohorts 2, 3 , and 4 ran for a full year. We collect recordings of all the synchronous meetings and we download a $\log$ of all the interactions on the asynchronous communication platform. We also interview participants about their FOLC experience.

In this paper we report solely on participants' selfreporting of community formation which comes from their interviews. We reserve analysis of interactions during synchronous meetings and on the asynchronous platform for a future paper. However, preliminary analysis of interactions on the asynchronous platform is published [7]. Cohort 4 finished in June 2017 and their interviews were not completed in time to be included in this paper. Therefore, we report on the twenty-four interviews collected from Cohorts 1-3.

The interviews of Cohort 1 participants were conducted approximately midway through their semester experience because they were our pilot group and we wanted to gauge how it was going before the semester ended. The interviews for all cohorts after Cohort 1 were conducted at the end of their oneyear experience. We were able to interview all participants from Cohorts 1 and 2. Cohort 3 started with ten participants, but three dropped out during the first semester. We were able to interview five of the seven participants who completed the full year experience and one of the drop-outs. Demographic information for the interviewees is shown in Table I. NFW participants are generally in their first few years of teaching in a tenure-track position. Compared to the NFW attendees overall, our FOLC interviewees were more likely to come from an undergraduate-only department (70\% vs 55\%) and more likely to be female (55\% vs $30 \%$ ) [8].

We conducted interviews using a semi-structured protocol. We asked participants about the different programmatic components of the FOLC, their overall assessment of the experience, and their impressions of community formation in their FOLC. Two of the authors (AL and MHD) first coded the interviews for any mention of community. Not only did participants talk about community when specifically asked about it, but it also arose in many other parts of their interviews. This initial pass at coding left us with 332 references to community in the interviews. We developed a sub-coding scheme
TABLE I. Gender composition (female/male) and department type of the interviewed participants, by cohort and overall. Physics/Astronomy departments are categorized as graduate degree granting $(\mathrm{G})$ or undergraduate only (UG).

\begin{tabular}{lcccc}
\hline \hline & $\begin{array}{c}\text { Cohort 1 } \\
\mathrm{n}=9\end{array}$ & $\begin{array}{c}\text { Cohort 2 } \\
\mathrm{n}=9\end{array}$ & $\begin{array}{c}\text { Cohort 3 } \\
\mathrm{n}=6\end{array}$ & $\begin{array}{c}\text { Total } \\
\mathrm{n}=24\end{array}$ \\
\hline Gender (F / M) & $6 / 3$ & $4 / 5$ & $3 / 3$ & $13 / 11$ \\
Institution (G / UG) & $4 / 5$ & $2 / 7$ & $1 / 5$ & $7 / 17$ \\
\hline \hline
\end{tabular}

after jointly reviewing the community excerpts from four of the interviews. We then tested the scheme on another five interviews and this led us to revise the scheme into its final form. We again separately coded community excerpts from five more interviews and then compared our work to ensure agreement on our code definitions. Then AL coded the remaining interviews, including revising the coding for the nine interviews where we had used a preliminary coding scheme.

\section{RESULTS}

\section{A. Did a community form?}

In the interviews participants were asked, "Does the FOLC feel like a community to you?" They were then prompted to explain their reasoning if it was not spontaneously offered. In asking this question, we did not define community for them. Almost all interviewed participants $(22 / 24,90 \%)$ said that their cohort did feel like a community. The two other interviewed participants described the community as "weak" or not "quite like" one yet. We note, however, both of these participants were from Cohort 1 which was interviewed after only a couple of months instead of at the end of a year.

When asked why it felt like a community, each participant $(\mathrm{n}=22)$ provided multiple reasons. The most prevalent reasons are listed in Table II. One-quarter of the participants who felt a community formed specifically mentioned that meeting first in-person at the NFW helped them start building those connections (which then grew during the FOLC experience).

Forty percent of the participants said that one of the reasons their cohort felt like a community was because all the members were in similar career positions and had shared interests. One person described it as, "feeling like you had a bunch of people in the same boat." Moreover, the cohorts were comprised "of people that are very interested in the teaching, as well as the other aspects of being a tenure-track." This is perhaps unsurprising as FOLC members are drawn from NFW participants who are mainly young faculty and obviously interested in teaching.

Almost half (45\%) of participants said the FOLC felt like a community because they got to know the other members' personalities and professional situations. One participant described, "We had a number of community sorts of things...as time went on we learned what people's expertise is...I guess 
it's sort of for me a sense that I know where people are coming from, I know their background...I think it sort of got to feel familiar after about, I don't know, three or four meetings online" In describing her sense of community another participant said, "At first I was just trying to remember who was who and where they were from, but for those of us who were able to attend regularly, by the end of the year I felt like I knew a little snippet of their lives and what they were doing and had some kind of a connection to them." From these two excerpts, one can also see that community took time to form.

Many participants $(45 \%)$ said that their cohort felt like a community because they were comfortable sharing aspects of their teaching lives with the group. A participant stated, "I feel safe posting a question or an idea or a confusion online, or even speak up when we are meeting online." These participants described being able to be vulnerable and honest with the FOLC about their teaching challenges.

Some participants (20\%) said they felt their FOLC cohort was a community because they cared about each other and felt obligated to answer each other's questions. One of the participants said, "I got invested in other people's success, and I really wanted to know how things they tried turned out, and I wanted to hear about how their students did or what kind of feedback they got on something. That made it feel very much like a community." Another participant talked about, "if there's questions up there [on the asynchronous platform] that nobody's answering and you know that somebody wants some help, how can I answer this question to help this person along. I think that kind of responsibility is kind of...You know that they're out there, they're working hard, and they're trying to improve and we're all trying to do that, and if there's something that I'm doing well how can I help other people, and hope that something I don't do well and they do well they can help me." In both excerpts participants are describing a desire to contribute to their cohort and help everyone succeed.

The most common reason for describing the FOLC as a community is that it was a group where members could ask questions, receive feedback, and share ideas regarding their teaching. This reason was given by $70 \%$ of the participants who said their FOLC felt like a community. As told by one of these participants, "I feel like I'm able to go to the group when I have a question or when I have a need and I might be able to get something back, but I also feel like there's an opportunity to give back to them as well. So the fact that it's kind of a two-way relationship and a conversation, that makes it very different than if it was just I'm going to email an expert in the field and hope they bother to answer me." Participants shared and received knowledge and advice with their cohort.

As mentioned, two of the interviewed participants said that their cohort did not feel "quite like" a community at the time of the interview. A few of the reasons they cited for the lack of community were the absence of some of the elements mentioned in Table II. Both people described a sense of not getting to know the other members at a deep enough level. One person said in-person interaction is important to him and he did not get a chance to meet many of his cohort members
TABLE II. Reasons given for why the FOLC felt like a community. (Percentages are out of those who said the FOLC did feel like a community, $n=22$. Percentages are rounded to the nearest $5 \%$ ).

\begin{tabular}{lc}
\hline \hline Reason & $\mathbf{N}(\boldsymbol{\%})$ \\
\hline Met in-person at New Faculty Workshop & $6(25 \%)$ \\
Were in similar situations/shared interests & $9(40 \%)$ \\
Got to know each other & $10(45 \%)$ \\
Could be vulnerable \& honest & $10(45 \%)$ \\
Felt responsibility to help each other & $5(20 \%)$ \\
Could ask questions, receive help, and/or share ideas & $15(70 \%)$ \\
\hline \hline
\end{tabular}

at the NFW. The other participant said that too much time passed in between the (biweekly) synchronous meetings.

\section{B. Did participants support each other's teaching efforts?}

In their interviews, almost all participants talked about how they asked for feedback and shared ideas about teaching with their cohort. Specifically, $90 \%$ of those interviewed described receiving and providing this type of support. (Not everyone described these interactions as a reason why the FOLC felt like a community, which is why the percent reported in Table II is smaller). The two people who did not report these types of interactions are the participants who were in the pilot cohort and did not feel the FOLC was a community yet. Examples of the "helping" behaviors are presented below.

One participant shared, "So going in[to the FOLC] I just sort of thought okay this will be another useful way of bouncing ideas off people and that probably is the single biggest thing I've gotten out of it, is just getting more perspectives." The FOLC, comprised of people from different schools, but who all cared about teaching, helped this participant gain new ideas to try in the classroom. This participant valued these interactions, calling the gained perspectives "the single biggest thing" he took away from his FOLC experience.

Additionally, FOLC members provided suggestions to each other when they had trouble implementing particular teaching techniques. For example, one participant relayed, "It was very frustrating because I tried it [Just In Time Teaching] last year, you know... and it didn't work out too well...And I was able to talk to some of the other FOLC members and they gave me some advice on what I could do." The FOLC provided support as participants tried the strategies introduced at the NFW, and this support came through the cohort's conversations about teaching.

Cohort Three had an activity at every meeting called "best and worst," in which everyone shared a highlight and a challenge from their teaching over the past week. In describing this activity one participant explained, "We all got to pat each other on the back when things were going well, and we all got to either just have some sympathy when things weren't going well or have some ideas as to how to improve." The group gave suggestions to each other when challenges arose and 
also provided moral support. This excerpt additionally shows that teaching support was not limited to difficult situations; participants congratulated each other on their successes.

\section{DISCUSSION}

We created our FOLCs to provide content and moral support for the sustained adoption of research-based instructional strategies. This extended support is rooted in the formation of a community devoted to improving its teaching. The results reported here indicate that community is formed in our FOLCs, although we cannot yet say how robustly this community continues past the official end of a FOLC.

The vast majority of interviewed participants, when directly asked whether the FOLC felt like a community, responded that it did (answering our first research question). However, statements indicate that community was not immediately present, although it did help if the participants met each other in-person at the NFW. It is interesting that while community did form and strengthen as the participants communicated virtually, it may still be important for their initial meeting to be in-person.

The reasons participants cited for why the FOLC felt like a community align with our hypotheses of what makes a community successful. First, they all described supporting each other with the technical/content aspects of teaching. Participants did indeed provide support for each other's teaching efforts (answering our second research question). They went to each other for feedback on strategies they were trying in the classroom and they shared ideas of new techniques to try. They also shared actual materials (i.e. rubrics, labs, clicker questions). Participants valued the diverse perspectives and ideas they received by interacting with the group.

Second, participants described encouraging each other and also celebrating each other's successes. A number of participants described a sense of responsibility they felt to help each other, and others framed this as being invested in the cohort's success. Even if a participant did not know the answer to a member's question, they showed empathy (acknowledging the challenge being described).

Underlying these two types of support are some of the other reasons why the FOLC felt like a community to most participants. Participants could share a failure from their classroom and workshop solutions because they felt comfortable being vulnerable with their cohort. Getting to know each other's personalities and professional situations provided context for the questions they asked each other. Finally, we started with participants who were all invested in teaching (evidenced by the fact that they joined the FOLC) and their shared interests and professional situations helped the cohort connect.

Unlike traditional FLCs, our FOLCs are disciplinary and non-local (draw members from different institutions). These differences do not prevent community formation, and it seems that having members in the same discipline (physics/astronomy) actually helps build the community. However, we need to further investigate how different teaching loads and institutional situations may slow or constrain community building.

\section{CONCLUSION}

The results from participant interviews are one indication that FOLCs are indeed building community, as they were designed to do. Moreover, this community is providing support to members as they work to improve their teaching. Future work will analyze interactions on the asynchronous platform and during synchronous meetings to form a more complete picture of how a sense of community forms for some (and why it doesn't for others) and the valued aspects of that community. We also plan to collect longitudinal data to see if community persists in the long-term and if it aids in sustained adoption of research-based instructional strategies.

\section{ACKNOWLEDGMENTS}

We would like to thank the facilitators of our FOLCs: Jeremy Bailin, Jessica Bickel, Zoe Boekelheide, Mary Bridget Kustusch, Gillian Ryan, and Adrienne Traxler. This project is funded by NSF grants DUE-0813481, DUE0121384, and DUE-9554738.
[1] Workshop for New Physics and Astronomy Faculty, http://www.aapt.org/Conferences/newfaculty/nfw.cfm, Accessed Oct 6, 2017.

[2] C. Henderson, M. Dancy, and M. Niewiadomska-Bugaj, Phys. Rev. ST Phys. Educ. Res. 8, 020104 (2012).

[3] C. Henderson and M. H. Dancy, Phys. Rev. ST Phys. Educ. Res. 3, 020102 (2007).

[4] M. D. Cox, New Dir. Teach. Learn. 2004, 5 (2004).

[5] A. Rundquist, J. C. Corbo, S. Chasteen, M. S. Martinuk, C. R. Henderson, and M. H. Dancy, in 2015 Physics Educa- tion Research Conference Proceedings (American Association of Physics Teachers, 2015).

[6] P. J. Baker and M. Zey-Ferrell, Teaching Sociology 12, 82 (1984).

[7] J. C. Corbo, A. Rundquist, C. R. Henderson, and M. H. Dancy, in 2016 Physics Education Research Conference Proceedings (American Association of Physics Teachers, 2016).

[8] S. V. Chasteen, R. Chattergoon, E. E. Prather, and R. Hilborn, in 2016 Physics Education Research Conference Proceedings (American Association of Physics Teachers, 2016). 